\title{
Locating Sensors in Concave Areas
}

\author{
Chen Wang, Li Xiao \\ Department of Computer Science and Engineering \\ Michigan State University \\ Email: \{wangchen, lxiao\}@cse.msu.edu
}

\begin{abstract}
In sensor network localization, multihop based approaches were proposed to approximate the shortest paths to Euclidean distances between pairwise sensors. A good approximation can be achieved when sensors are densely deployed in a convex area, where the shortest paths are close to straight lines connecting pairwise sensors. However, in a concave network, the shortest paths may deviate far away from straight lines, which leads to erroneous distance estimation and inaccurate localization results. In this paper, we propose an improved multihop algorithm which can recognize and filter out the erroneous distance estimation, and therefore achieve accurate localization results even in a concave network.
\end{abstract}

\section{INTRODUCTION}

It is important to determine sensors' locations because it provides fundamental support for many location-aware protocols[1][2][3] and applications[4]. A simple approach is to infer a sensor's position through GPS, which measures distances from a sensor to multiple reference points in Satellites and calculates the sensor's position through triangulation computation. However, due to the low cost design constraint, it is prohibitive to equip GPS receivers in all sensors. A compromised solution is to deploy GPS receivers to a few sensors which are defined as beacons. The rest of sensors infer their positions based on their relative distances to those beacons. Based on this model, the sensor localization problem can be formalized as follows. Given a network graph $G=$ $\left(V_{m} \cup V_{n}, E\right)$, the vertex set $V_{m}$ defines the beacons set, the vertex set $V_{n}$ defines the sensor set whose coordinates are unknown, and the edge set $E$ defines all the measurable distances between pairs of vertex $(i, j)$ where $i, j \in V_{m} \cup V_{n}$. The sensor localization is to recover coordinates of the vertex in the set $V_{n}$ under the constraints of edge set $E$ and beacon set $V_{m}$.

Despite its simple description in mathematics, sensor localization is a challenging task in engineering which imposes tight design constraints on sensor nodes with low costs, power saving and small dimensions. Under such constraints, current available distance measurement techniques based on ultrasound can only achieve accurate results in short-range, such that beacons are not globally accessible to all sensors especially when beacons are sparsely distributed. Consequently, the simple triangulation algorithm cannot be directly applied to locate all sensors because some sensors may not have sufficient beacons available as their immediate neighbors.

To overcome the limitation of short-range distance measurements, multihop based approaches were proposed to infer distances between any pair of sensors (including beacons) by approximating the lengths of the shortest paths to the Euclidean distances. The localization accuracy of multihop based approaches are built on the basis that the Euclidean distances between pairwise sensors can be well approximated by the lengths of the shortest paths. Such an approximation is achievable only when the shortest paths are close to straight lines, which requires sensor nodes are uniformly and densely distributed in a convex area. Although the uniform and dense distribution can be achieved through controlled deployment, it cannot be guaranteed that sensors are deployed in a convex area. A typical example is in habitat monitoring, sensors are deployed to complex areas such as valleys or rivers (Fig. 1) which may have concave shapes. The other scenario is that sensors are deployed in streets of urban areas where sensors may be separated from each other by buildings which results in concave network topologies. In such cases, the lengths of the shortest paths may not reflect the Euclidean distances correctly, because the shortest paths between some pairwise sensors have to detour along the concave areas (for instance $\mathrm{C}$ shape or $\mathrm{S}$ shape shown in Fig. 2 and Fig. 3) and cannot be close to a straight line no matter how densely sensors are deployed. Since concave scenarios in the real world such as rivers are the combinations of $\mathrm{C}$ shapes and $\mathrm{S}$ shapes, we choose the $\mathrm{C}$ shape and the $S$ shape as the representatives to be discussed in this paper.

Previous work[5][6] has shown that the localization results of Multidimensional Scaling (MDS) and triangulation are severely corrupted by the large errors of incorrect distance measurements which are distorted by concave shapes. While an intuitive solution is that those distorted distance measurements should be filtered out and not involved in the localization, it is impossible to recognize whether a single distance measurement is affected by concave shapes or not due to the lack of a global geometrical view of a network. However, our work shows that it is possible to filter out the impact of distorted distance measurements when multiple distances from beacons to a sensor are available. Our approach is different from previous work in that the distorted distance measurements are directly filtered out and not involved in the localization any more, and therefore their impact on the final localization results are minimized.

The remainder of the paper is organized as follows. Section II introduces previous work in sensor localization. Section III describes how the distorted distance measurements are filtered out in our improved Multihop algorithm. Section IV evaluates the performance of our approach through the comparison with 


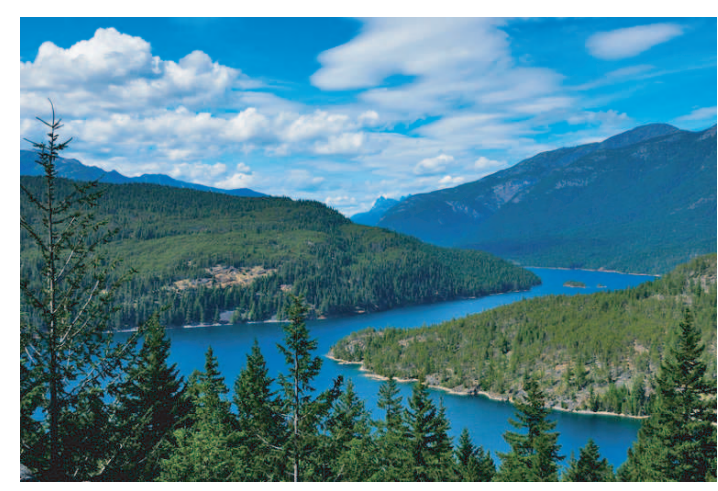

Fig. 1. Sensors are deployed along a river



Fig. 2. Sensors are deployed in a $\mathrm{C}$ shape area

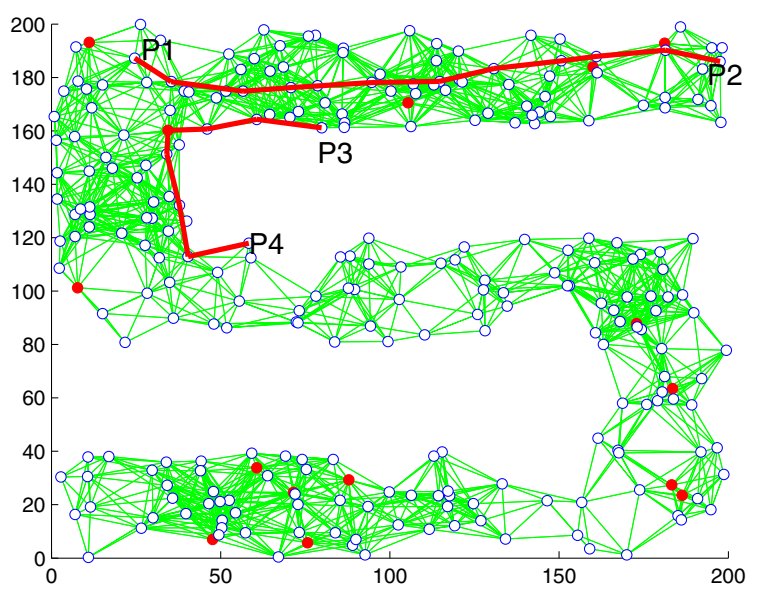

Fig. 3. Sensors are deployed in a S shape area previous work. We extend the improved Multihop algorithm to the iterative approach in section $\mathrm{V}$. The conclusion is made in Section VI.

\section{STATE OF THE ART}

As described in Section 1, senor localization is to recover sensors' coordinates under the edge length constraints. Two steps are involved in this process: 1) the edge lengths are obtained through in-network distance measurements between neighboring sensors; 2)coordinates of sensors are calculated by localization algorithms based on measured distances.

Due to the cost, dimension and energy design constraints of sensors, currently available in-network distance measurements based on radio received signal strength(RSS) or ultrasound are either error-prone[7][8] or short-range, which raises challenges to design an effective localization algorithm. Many algorithms have been proposed to locate sensors by overcoming the limitation of incapable distance measurements. According to whether actual distances are involved in the localization, we divide those algorithms to two categories: area-based algorithms and distance-based algorithms.

\section{A. Area-based algorithms}

In area-based algorithms, actual distance values are not involved in the localization algorithms, and the position of a sensor is estimated by picking up a point within an area. A typical example is the Centroid approach[9], which estimates a sensor's position as the centroid of the polygon formed by beacons that are within the radio transmission range of the sensor.

APIT[10] approach further improves the localization accuracy by utilizing the redundancy of available beacons, from which 3 beacons are selected out to form a triangle. The APIT approach can determine if a sensor is within the triangle by comparing the beacons' RSS with immediate neighbors. It is possible to obtain multiple triangles from different beacon combinations, and a set of triangles containing the sensor can be selected out. The sensor's position can be pinpointed to the intersection of all the containing triangles, which can be a small area when multiple triangles are involved in the intersection. As a result, the APIT approach can achieve better localization result than the basic Centroid approach.

\section{B. Distance-based algorithms}

Distance-based algorithms estimate sensors' positions from pairwise node distances, which are obtained through the media of radio signals or ultrasound with different measurement accuracy. All the distance-based algorithms recover coordinates of sensor nodes from distance constraints, such that pairwise node distances calculated from recovered coordinates are consistent with correspondent measured distances.

The basic distance-based algorithm is the multilateration, which is applicable when distances from a sensor to multiple beacons are available. To tolerate distance measurement errors, least squares fitting is proposed to minimize the difference 
between calculated distances and measured distances from the sensor to all available beacons:

$$
\widehat{\mathbf{p}}=\arg \min _{\mathbf{p}} \sum\left(\left|\mathbf{p}-\mathbf{p}_{i}\right|-\widehat{d}_{i}\right)^{2}
$$

where $\mathbf{p}$ is the sensor's position to be estimated, $\mathbf{p}_{i}$ are beacons' positions, $\left|\mathbf{p}-\mathbf{p}_{i}\right|$ are calculated distances and $\widehat{d}_{i}$ are measured distances.

As we mentioned before, in-network distance measurements between pairwise sensors are often short-range. The basic multilateration algorithm may fail for some sensors due to insufficient beacons available as their immediate neighbors. Two possible solutions to overcome the limitation of shortrange measurement are recursive approaches and mutihop based approaches.

Recursive approaches[11][7][12] repeatedly apply the basic multilateration algorithm by converting sensors to beacons after their positions are determined. In recursive approaches, "converted" beacons can be propagated from an area which is close to the "starting" beacons to an area where the "starting" beacons are inaccessible. This makes it possible for sensors faraway from "starting" beacons to locate themselves with the aid of "converted" beacons. One problem of recursive approaches is that the localization error may accumulate and the final result may be severely distorted. To minimize the jeopardy of accumulated errors, recursive approaches are usually built on the basis of accurate distance measurements such as time of flight(ToF) of ultrasound. It is also pointed out in [12] to avoid large errors such as flip over when beacons are distributed closely to a straight line.

By approximating the length of the shortest path to the Euclidean distance between pairwise nodes, multihop based approaches[9][13][14][15] can infer distances between any pair of nodes in a connected network, hence all beacons are accessible to each sensor. Consequently, each sensor can locate itself through the basic multilateration algorithm. Multihop based approaches can only provide coarse localization results due to their approximate distance estimation. However, multihop based approaches are low-cost solutions because they suggest to reuse the communication radio signals to infer pairwise node distances. The low-cost character makes multihop based approaches ideal candidates for applications which have tight restriction on cost and dimension while less demand on localization accuracy.

When a global view of all pairwise node distances is available, coordinate assignment can be achieved through centralized localization algorithms such as Mulitdimensional Scaling (MDS) [5][16][17] and Semidefinate Programming (SDP)[18][19]. Both approaches use optimization algorithms to search for coordinate assignment such that the distance constraints can be best fit. For instance, the MDS is to solve the following optimization problem which minimize the difference between all calculated distances and measured distances:

$$
\widehat{P}=\arg \min _{P} \sum_{i, j \in V}\left(\left|\mathbf{p}_{i}-\mathbf{p}_{j}\right|-\widehat{d}_{i j}\right)^{2} .
$$

Here, $P$ is the position matrix which contains all the sensors' positions to be estimated. $\left|\mathbf{p}_{i}-\mathbf{p}_{j}\right|$ is the calculated distance, $\widehat{d}_{i j}$ is the measured distance between sensor $i$ and $j$ and $V$ is the vertex set representing all sensors. It is notable that the multihop approaches are also suggested in MDS[16] to infer distances between any pair of sensors since the classic MDS requires distance knowledge of all pairs of nodes.

Both the multilateration and MDS are built on the basis of least squares fitting, which fits the calculated distances to measured distances. The least squares fitting is based on the belief that all the distance measurements are close to their true values and have equal error distributions. However, when sensors are deployed in a concave environment, some of the distances estimated by the multihop based approach may deviate far away from their true values because the shortest paths have to detour along the concave shapes and deviate from straight lines. If we still use the least squares fitting algorithm to equally fit all the measurements, the final positioning results will be deteriorated by those "bad" distance measurements. To address this issue in multihop algorithms, two approaches have been proposed before.

It is suggested in [20] to use the four nearest beacons instead of all of them in the multilateration localization. This is based on the observation that the distances from a sensor to the nearest beacons will be less affected by the concave shapes. However, it is still possible that the shortest path to the nearest beacon is affected by the concave shapes. We will compare this approach with our approach in detail in the coming sections.

Proximity-distance map(PDM) is proposed in [6] to approximate lengths of the shortest paths to Euclidean distances correctly in anisotropic networks. In PDM, each sensor is assigned a coordinates in M-dimensional embedding space defined by the lengths of the shortest paths from sensors to all $M$ beacons:

$$
\mathbf{p}_{i}=\left[p_{i 1}, \ldots p_{i M}\right]^{T},
$$

where $p_{i j}$ is the length of the shortest path from the $i t h$ sensor to $j$ th beacon. The objective of PDM is to find out sensors' coordinates in M-dimensional embedding space defined by Euclidean distances from sensors to all $M$ beacons:

$$
\mathbf{l}_{i}=\left[l_{i 1}, \ldots l_{i M}\right]^{T},
$$

where $l_{i j}$ is the Euclidean distance from the $i t h$ sensor to $j t h$ beacon. When the Euclidean distances from a sensor to all the beacons are available, the multilateration such as least squares fitting can be used to calculate sensor's location based on those Euclidean distances. PDM assumes there exists a linear transform between $\mathbf{p}_{i}$ and $\mathbf{l}_{i}$ such that $\mathbf{l}_{i}=T \mathbf{p}_{i}$. The linear transform $T$ can be learned from beacons, where beacons' coordinates of both $\mathbf{p}_{i}$ and $\mathbf{l}_{i}$ are known.

The intuition of PDM is that the topology character of the entire network can be well represented by the beacons, since they are uniformly distributed in the network. Therefore, the linear transform $T$ learned from beacons can be also applied to other sensors to transform their coordinates $\mathbf{p}_{i}$ to coordinates $\mathbf{l}_{i}$ defined by Euclidean distances. In this paper, 


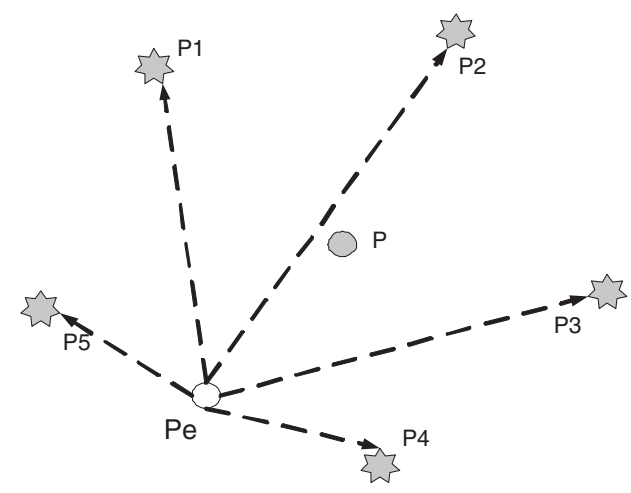

Fig. 4. The estimated position $P e$ is push away from the true position $P$ by the incorrect distance measurement between $P$ and $P_{2}$

we are trying to improve the least squares fitting algorithm in the multihop scenario. It is possible to apply our improved Multihop algorithm based on the Euclidean distances obtained by the PDM.

\section{IMPROVED MULTIHOP APPROACH}

In this section we will introduce our improved multihop ( $\boldsymbol{i}$ Multihop) approach and explain how it can be extended to concave areas. To facilitate our discussion, we define symbols in the table below.

$\begin{array}{ll}\text { Symbol } & \begin{array}{l}\text { Defination } \\ \text { position of the sensor to be estimated }\end{array} \\ \left|\mathbf{p}-\mathbf{p}_{i}\right| & \begin{array}{l}\text { Euclidean distance calculated from a sen- } \\ \text { sor's position } \mathbf{p} \text { to a beacon's position } \mathbf{p}_{i}\end{array} \\ \widehat{d}_{i} & \begin{array}{l}\text { measured distance between a senor and the } \\ \text { ith beacon } \\ \text { true Euclidean distance between a sensor } \\ d_{i}\end{array} \\ & \text { and the } i t h \text { beacon }\end{array}$

Before we detail our $i$-Multihop algorithm, we will review the original multihop approach and investigate why it fails in concave areas.

\section{A. Multihop algorithm: distance fitting approach}

The key idea of multihop approaches is to discover a sensor network's geometry structure from its communication network topology. In multihop approach, a sensor network is viewed as a connected graph $G=(V, E)$, where $V$ is the vertex set representing sensors and $E$ is the edge set representing links between a pair of sensors which are within radio transmission range. Multihop approaches infer the distance between a pair of sensors by approximating the length of the shortest path to the Euclidean distance. the length of the shortest path between vertex $m$ and $n$ is calculated as $L_{m n}=\sum l_{i}$, where $l_{i}$ are the lengths of intermediate edges included in the shortest path. The value of $l_{i}$ can be inferred from RSS which attenuates exponentially when the transmission distance is increased.

In the cases where RSS value is not available, multihop approaches infer the length of the shortest path from the average length per hop, which can be sampled by beacons as follows.
1) Distances $D_{i j}$ between any pair of beacons can be evaluated from their known coordinates.

2) The number of hops $H_{i j}$ of the shortest path between pairs of beacons can be inferred from the Dijkstra or Distance Vector algorithm.

3) The average length per hop to the ith beacon can be calculated as

$$
h_{i}=\frac{\sum_{j \in V_{m}} D_{i j}}{\sum_{j \in V_{m}} H_{i j}},
$$

where $V_{m}$ is the beacon set.

When the average length per hop is available, the length of the shortest path from a sensor to the $i t h$ beacon can be calculated as

$$
L_{i}=h_{i} \times H_{i},
$$

where $H_{i}$ is the number of hops of the shortest path from the sensor to the ith beacon.

The accuracy of multihop approaches is built on the assumption that the shortest path between a pair of sensors is close to a straight line, which is possible as long as the following assumption hold:

1) Sensors and beacons are densely and uniformly distributed.

2) The network is maintained as a connected graph.

3) The deployed area has a convex shape.

The uniform and dense distribution of sensors can be achieved through a carefully controlled deployment. How to maintain $k$-connectivity of a network by selecting a proper transmission power is well studied in [21][22]. Therefore, it is not difficult to hold the first two assumptions. However, the third assumption cannot be guaranteed in practice, since the shapes of deployed areas are often out of human being's control.

As we pointed out before, the concave shape has severe impact on distance estimation of multihop approaches. Although a good approximation between the length of the shortest path and the Euclidean distance can still be achieved in some scenario (for instance $P_{1} P_{2}$ shown in Fig. 2), the lengths of the shortest paths may differ significantly from the Euclidean distances between pairwise nodes. This is because the shortest paths may be distorted by the concave area and cannot be close to a straight line. Such an example is shown as $P_{3} P_{4}$ in Fig. 2. To distinguish the distorted distance estimation from the rest, we divide the distances estimated by the multihop approach into two categories: one is incorrect distance measurements which are distorted by the concave shape, the other is correct distance measurements which are not affected by the concave shape. Previous work[5][6] has shown that the localization results of MDS and mltilateration are severely corrupted by the large error of incorrect distance measurements.

As we discussed before, to offset the inaccuracy of distance measurements, the least squares fitting is suggested in multilateration to estimate sensors' positions by minimizing the difference between the calculated distances and measured distances as Eqn (1). Based on the belief that all the distance measurements $\widehat{d}_{i}$ have the same error distribution and are close 
to their true values $d_{i}\left(\widehat{d}_{i} \approx d_{i}\right)$, the least squares fitting tries to fit each measured distance equally. Thus, the final estimated position is the averaged result of all measurements and the impact of an individual measurement error is reduced. However, the assumption $\widehat{d}_{i} \approx d_{i}$ does not hold in a concave area, since the incorrect distance measurement has much larger error than the rest. The final localization result is corrupted by fitting to the incorrect distance measurements. An example is shown in Fig. 4.

\section{B. Multihop algorithm: the four nearest beacons}

To eliminate the impact of concave shapes, it is proposed in [20] which uses the 4 nearest beacons instead of all of them. The intuition is that the shortest path from a sensor to the nearest beacon may be less affected by concave shapes. To facilitate our presentation, we denote this algorithm as the $n$-Multihop algorithm. The $n$-Multihop algorithm has two potential limitations.

1) The shortest path to the nearest beacon does not necessarily mean it is not affected by the concave shape. An example is shown in Fig. 3 where the path $P_{1} P_{2}$ is longer than $P_{3} P_{4}$, while the former is less affected by the concave shape and more close to its Euclidean distance.

2) By only using 4 beacons, some of the good distance measurements are eliminated from the localization, and therefore the redundancy of available beacons is sacrificed.

Now we start to detail our $i$-Multihop algorithm as below.

\section{C. i-Multihop algorithm: upper bound approach}

To facilitate the discussion, we first assume the in-network distance measurements between immediate neighbors are accurate, thus the mismatch between the shortest paths and straight lines connecting pairwise sensors is the only source of the distance measurement error. We will relax this assumption in later discussion. Based on this assumption, we can have the following observation:

Observation 1: All the measured distances $\widehat{d}_{i}$ are no less than their true value $d_{i}\left(\widehat{d}_{i} \geq d_{i}\right)$, because the length of the shortest path is always longer than the Euclidean distance of the straight line connecting pairwise nodes. Especially, the incorrect distance $\widehat{d}^{\prime}{ }_{i}$ distorted by the concave shape is much larger than its true value $d^{\prime}{ }_{i}\left(\widehat{d}_{i}^{\prime}{ }_{i} \gg d_{i}^{\prime}\right)$, because the shortest path deviates significantly from the straight line.

Based on the observation above, we model sensor localization in concave areas as below.

Model 1: Given a network graph $G=\left(V_{m} \cup V_{n}, E_{s} \bigcup E_{t}\right)$, the vertex set $V_{m}$ defines the beacons set; the vertex set $V_{n}$ defines the sensor set whose coordinates are unknown; the edge set $E_{s}$ defines all the correct distance measurements $\widehat{d}_{i} \geq$ $d_{i}$; and $E_{t}$ defines all the incorrect distance measurements $\widehat{d}_{i}^{\prime} \gg d_{i}^{\prime}$. The sensor localization is to filter out the incorrect distance measurements $E_{t}$ and recover coordinates of the vertex set $V_{n}$ under the constraints of correct measurements $E_{s}$ and beacon set $V_{m}$.

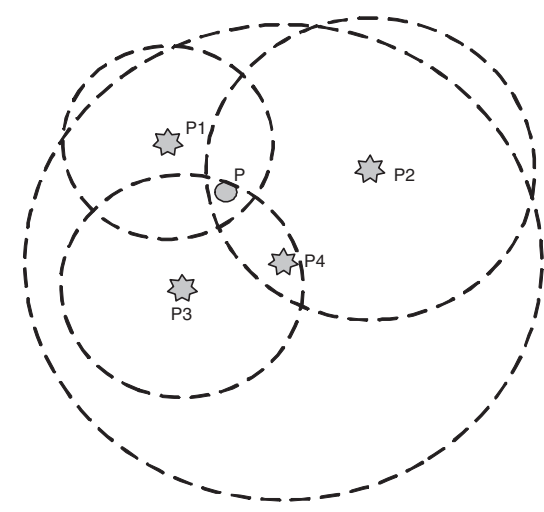

Fig. 5. Sensor $P$ is tightly constrained in the intersection of the circular regions $P_{1}, P_{2}$ and $P_{3}$

The challenge is how to recognize the incorrect distance measurements from the rest in the model where incorrect distance measurements are mixed together with correct distance measurements, which is impossible to be achieved by observing an individual distance measurement alone. However, we show that it is possible to eliminate the impact of incorrect distance measurements from the final localization result when multiple distance measurements are available. Instead of fitting distance measurements, we use upper bound constraints to locate sensors as below.

$$
\begin{gathered}
\widehat{\mathbf{p}}=\arg \min _{\mathbf{p}} \sum\left|\mathbf{p}-\mathbf{p}_{i}\right|^{2} \\
\text { subject to }\left|\mathbf{p}-\mathbf{p}_{i}\right| \leq \widehat{d}_{i}
\end{gathered}
$$

Remark 1: The algorithm described in Eqn (2) can filter out incorrect distance measurements and achieve accurate localization results if and only if the number of correct distances is no less than 3 .

Based on our assumption $\widehat{d}_{i} \geq d_{i}$ we have distance measurement error $\delta_{i}=\widehat{d}_{i}-d_{i} \geq 0$. Without losing generality, for all the distances measurements between the senor and beacons, we assume $\delta_{1} \leq \delta_{2} \leq \ldots \leq \delta_{m} \ll \delta_{m+1} \leq \delta_{m+2} \ldots \leq \delta_{n}$. Here, $\delta_{i}(1 \leq i \leq m)$ are small errors of correct distance measurements, and $\delta_{i}(m+1 \leq i \leq n)$ are large errors of incorrect distance measurements.

Since $\widehat{d}_{i} \geq d_{i}$, we have $\mathbf{p} \in C_{i}$, where $C_{i}$ is the circular region with origin $\mathbf{p}_{i}$ and radius $\widehat{d}_{i}$. We define the area of $C_{i}$ as $S_{i}$, which represents the uncertainty of the estimated position $\widehat{p}$. Based on the knowledge of $\widehat{d}_{i} \geq d_{i}$, we have $\left|\mathbf{p}-\mathbf{p}_{i}\right| \leq \widehat{d}_{i}$. The probability of estimated position $\mathbf{p}$ follows the uniform distribution:

$$
p(\mathbf{p})= \begin{cases}1 / S_{i} & , \text { if }\left|\mathbf{p}-\mathbf{p}_{i}\right| \leq \widehat{d}_{i} \\ 0 & , \text { otherwise }\end{cases}
$$

When $S_{i}$ becomes smaller, the probability density of $p(\mathbf{p})$ is increased. Thus, the uncertainty of the estimated position $\widehat{\mathbf{p}}$ is decreased. When multiple distance measurements are available, the true position of node $\mathbf{p}$ should be in the intersection of all circular regions $C_{i}$, i.e. $\mathbf{p} \in I=\bigcap_{1 \leq i \leq n} C_{i}$, and the probability of $\mathbf{p}$ follows: 


$$
p(\mathbf{p})= \begin{cases}1 / S(I) & , \text { if } \mathbf{p} \in I \\ 0 & , \text { otherwise }\end{cases}
$$

Here, the area $S(I)$ of intersection region $I$ represents the uncertainty of the final estimation result. If $\delta_{i} \rightarrow 0, S(I) \rightarrow 0$, the estimated position $\widehat{\mathbf{p}}$ can be accurately pinpointed to its true position $\mathbf{p}$.

Let $S_{t}=\bigcap_{1 \leq i \leq m} C_{i}$. We have $S(I) \leq S_{t}$, which means the uncertainty will not be increased when incorrect distance constraints are added in the localization. Therefore, the incorrect distance measurements will not deteriorate the final localization result. The fundamental reason why the upper bound approach can tolerate the incorrect distance measurement is that its assumption $\widehat{d_{i}} \geq d_{i}$ is consistent with the observation $\widehat{d}_{i} \gg d_{i}$, while the assumption $\widehat{d}_{i} \approx d_{i}$ of the original distance fitting algorithm is inconsistent with the observation $\widehat{d}_{i} \gg d_{i}$.

An example of upper bound approach is shown in Fig. 5, where the distance measurement between $P$ and $P_{4}$ is much longer than its true value, which results in the large circle constraint. However, the incorrect distance measurement between $P$ and $P_{4}$ has no impact on the final estimation result, since sensor $P$ is tightly constrained by the constrained circular regions of $P_{1}, P_{2}$ and $P_{3}$.

The previous work[18][23] also suggests to use the upper bound constraints to locate sensors. Instead of using the local optimization where only distance to immediate neighboring beacons are used, the work [18] uses the global optimization of semidefinite programming. However, all approaches suffer the same problem, which requires that beacons are placed on the outside boundary of deployed area. Otherwise, the estimated positions will collapse toward the center. Such a phenomena have been observed in previous work[19][20]. Below is a formalized description of the problem.

Problem 1: Given beacons $\mathbf{p}_{i}$, there exists a polygon region $P$ with all the vertices of $\mathbf{p}_{i}$. if $\mathbf{p}$ is not within the polygon $P$ $(\mathbf{p} \notin P)$, the position $\widehat{\mathbf{p}}$ estimated by upper bound approach will collapse toward the $P$.

An example is shown in Fig. 6, where $\mathbf{P} \notin \triangle \mathbf{P}_{1} \mathbf{P}_{2} \mathbf{P}_{3}$. The sensor is constrained by a large intersection area, thus has high uncertainty. Position $P e$ estimated by the upper bound algorithm of Eqn (2) is attracted towards the $\triangle \mathbf{P}_{1} \mathbf{P}_{2} \mathbf{P}_{3}$ and deviates from its true position. This is contrast to previous example in Fig. 5, where sensor $\mathbf{P}$ is tightly constrained in a small intersection because beacons are distributed around the sensor.

To solve the collapse problem, we propose our solution as below.

\section{D. i-Multihop algorithm: hybrid approach}

In this section, we assume that sensors are densely distributed, thus the correct distance measurements, which are not affected by the concave shape, are close to their true values. Based on this assumption, we have the observation below.

Observation 2: All the measured distances $\widehat{d}_{i}$ are no less than its true value $d_{i}\left(\widehat{d}_{i} \geq d_{i}\right)$. For all the correct distance



Fig. 6. Collapsed result of the upper bound approach

measurements $\widehat{d}_{i}$, we have $\widehat{d}_{i} \approx d_{i}$. For incorrect distance measurements, we have $\left(\widehat{d}^{\prime}{ }_{i} \gg d_{i}^{\prime}\right)$.

Based on the Observation 2, we model the sensor localization as below.

Model 2: Given a network graph $G=\left(V_{m} \bigcup V_{n}, E_{s} \bigcup E_{t}\right)$, the vertex set $V_{m}$ defines the beacons set; the vertex set $V_{n}$ defines the position unknown sensor set; the edge set $E_{s}$ defines all the correct distance measurements $\widehat{d}_{i} \geq d_{i} \& \widehat{d}_{i} \approx d_{i}$; and $E_{t}$ defines all the incorrect distance measurements $\widehat{d}^{\prime}{ }_{i} \gg d_{i}^{\prime}$. The sensor localization is to recover coordinates of vertex set $V_{n}$ by filtering out the incorrect distance measurements $E_{t}$ and fitting the correct distance measurements.

Based on the Model 2, we propose the localization algorithm below.

$$
\begin{aligned}
\widehat{\mathbf{p}}= & \arg \min _{\mathbf{p}} \sum\left(\left|\mathbf{p}-\mathbf{p}_{i}\right|-d_{i}\right)^{2} \\
& \text { subject to }\left|\mathbf{p}-\mathbf{p}_{i}\right| \leq \widehat{d}_{i}
\end{aligned}
$$

Remark 2: The algorithm described in Eqn (3) can filter out the incorrect distance measurements, and the final localization results will not collapse even when the sensor is not contained in the polygon formed by beacons.

The hybrid algorithm described in Eqn (3) combines the advantage of upper bound constraints and distance fitting. First, it uses the upper bound constraints to filter out the impact of incorrect distance measurements and pinpoint the estimated position to the intersection constrained by correct distance measurements. Second, it uses the distance fitting to fit correct distance measurements, which pushes the estimated position $\widehat{\mathbf{p}}$ toward its true position $\mathbf{p}$ and the final estimated position is not affected by the layout of beacons.

To facilitate the optimization computing, the algorithm in Eqn (3) can be simplified as below.

Since $\left|\mathbf{p}-\mathbf{p}_{i}\right| \leq \widehat{d}_{i}$, we have $\left|\mathbf{p}-\mathbf{p}_{i}\right|-\widehat{d}_{i} \leq 0$, thus $\widehat{d_{i}}-\left|\mathbf{p}-\mathbf{p}_{i}\right| \geq 0$. The objective function (3) can be simplified as:

$$
\widehat{\mathbf{p}}=\arg \min _{\mathbf{p}} \sum\left(\widehat{d}_{i}-\left|\mathbf{p}-\mathbf{p}_{i}\right|\right),
$$

which is equivalent to

$$
\widehat{\mathbf{p}}=\arg \min _{\mathbf{p}}-\sum\left(\left|\mathbf{p}-\mathbf{p}_{i}\right|\right) .
$$

Since $\left|\mathbf{p}-\mathbf{p}_{i}\right| \geq 0$, we can rewrite the objective function as:

$$
\widehat{\mathbf{p}}=\arg \min _{\mathbf{p}}-\sum\left(\left|\mathbf{p}-\mathbf{p}_{i}\right|\right)^{2}
$$


The final objective function is described as below.

$$
\begin{gathered}
\widehat{\mathbf{p}}=\arg \min _{\mathbf{p}}-\sum\left(\left|\mathbf{p}-\mathbf{p}_{i}\right|\right)^{2} \\
\text { subject to }\left|\mathbf{p}-\mathbf{p}_{i}\right| \leq \widehat{d}_{i},
\end{gathered}
$$

which is equivalent to the objective function below.

$$
\begin{gathered}
\widehat{\mathbf{p}}=\arg \max _{\mathbf{p}} \sum\left(\left|\mathbf{p}-\mathbf{p}_{i}\right|\right)^{2} \\
\text { subject to }\left|\mathbf{p}-\mathbf{p}_{i}\right| \leq \widehat{d}_{i}
\end{gathered}
$$

The intuition of the algorithm above is that the estimated position is pushed far away from beacons, thus towards the outside constrained boundaries. The final optimization result is that the estimated position is limited in the intersection area of circular constrained regions (upper bound constraints) and pushed towards the constrained boundaries (distance fitting), which is the intent of the hybrid approach.

\section{E. i-Multihop algorithm: final version}

In this section, we will relax the assumption that the innetwork distance measurements between immediate neighbors are accurate. Without this assumption, distances estimated from the lengths of the shortest paths will have two error sources: one is the measurement error between immediate neighbors, the other is incurred when the shortest path is not close to a straight line. Under this circumstance, it is possible that some of the distance measurements are less than their true values. This happens in correct distance measurements where the shortest path is very close to a straight line and the measured distance of each segment is less than its true values, such that the length of the shortest path is less than the Euclidean distance due to the error accumulation of each segments. Based on this analysis, we have the following observation.

Observation 3: All the correct distance measurements $\widehat{d}_{i}$ is close to (either larger or less than) its true value $d_{i}\left(\widehat{d}_{i} \approx d_{i}\right)$. All the incorrect distance measurements $\widehat{d}_{i}$ is larger than its true value $d_{i}\left({\widehat{d^{\prime}}}_{i} \gg d_{i}^{\prime}\right)$.

Based on this observation, the sensor localization is remodeled as below.

Model 3: Given a network graph $G=\left(V_{m} \bigcup V_{n}, E_{s} \bigcup E_{t}\right)$, the vertex set $V_{m}$ defines the beacons set; the vertex set $V_{n}$ defines the position unknown sensor set; the edge set $E_{s}$ defines all the correct distance measurements $\widehat{d}_{i} \approx d_{i}$; and $E_{t}$ defines all the incorrect distance measurements $\widehat{d}_{i}^{\prime} \gg d_{i}^{\prime}$. The sensor localization is to recover coordinates of vertex set by filtering out the incorrect distance measurements $E_{t}$ and fitting the correct distance measurements.

The hybrid approach with the upper bound constraints will not work in the model 3, because incorrect distance measurements may be less than their true values such that the constrained circular regions cannot intersect with each other. In such a case, no feasible position exists to satisfy all the upper bound constraints and the objective function will not find out a suitable solution.
To solve the problem above, we add slack variables $\varepsilon_{i}$ to the hybrid approach to get our final version of the $i$-Multihop algorithm.

$$
\widehat{\mathbf{p}}=\arg \min _{\mathbf{p}} \sum\left(\widehat{d}_{i}+\varepsilon_{i}-\left|\mathbf{p}-\mathbf{p}_{i}\right|\right)^{2}+k \sum \varepsilon_{i}
$$

$$
\text { subject to }\left|\mathbf{p}-\mathbf{p}_{i}\right| \leq \widehat{d_{i}}+\varepsilon_{i},
$$

where $k$ is the weight coefficient which is set to a large value $\left(10^{6}\right.$ in our computation). Due to the large value of the weight coefficient $k$, the second apart $k \sum \varepsilon_{i}$ has much higher priority to be minimized than the first part, which means the slack variable $\varepsilon_{i}$ has higher priority to be minimized than the difference between calculated distance $\left|\mathbf{p}-\mathbf{p}_{i}\right|$ and the measured distance $\widehat{d}_{i}$.

The intuition behind the objective function (5) can be described as follows. For those distance measurements which are less than their true values, the slack variables $\varepsilon_{i}$ can increase the upper bound to minimum extent such that the summary of the measured distance $\widehat{d}_{i}$ and the slack variable $\varepsilon_{i}$ is greater than the true value $d_{i}$. The consequence is that all the circular region constraints intersect into an non-empty set which contains a feasible solution for the objective function. Through this way, the problem is transformed to Model 2 where all the upper bound constraints are larger than the true distances. Therefore, we can use the similar approach as Model 2 to locate sensors by filtering out incorrect distance measurements and fitting correct distance measurements.

Again, we simplify the objective function (5) to facilitate our optimization computation as below.

$$
\begin{aligned}
\widehat{\mathbf{p}}= & \arg \min _{\mathbf{p}}-\sum\left(\left|\mathbf{p}-\mathbf{p}_{i}\right|\right)^{2}+k \sum \varepsilon_{i} \\
& \text { subject to }\left|\mathbf{p}-\mathbf{p}_{i}\right| \leq \widehat{d}_{i}+\varepsilon_{i}
\end{aligned}
$$

\section{F. Average length per hop}

In the case where distance measurements between immediate neighbors are unavailable, multihop algorithm estimate distances by multiplying the number of hops of the shortest path with the average length per hop. Here, the average length per hop to the ith beacon can be sampled as below.

$$
h_{i}=\frac{\sum_{j \in V_{m}} D_{i j}}{\sum_{j \in V_{m}} H_{i j}}
$$

where $D_{i j}$ is the Euclidean distance from the $j t h$ beacon to the $i t h$ beacon, and $H_{i j}$ is the number of hops from the $j t h$ beacon to the $i t h$ beacon.

However, the average length per hop calculated as above can be severely affected by the concave shapes. Because the shortest path between beacons may also be distorted by the concave shape and deviate far away from a straight line, the actual length $L_{i j}$ of the shortest path will be much longer than the Euclidean distance $D_{i j}$ calculated from the coordinates of the beacons. Therefore, the true value of the average length per hop $L_{i j} / H_{i j}$ is much larger than the value estimated from $D_{i j} / H_{i j}$. This will make the average value 

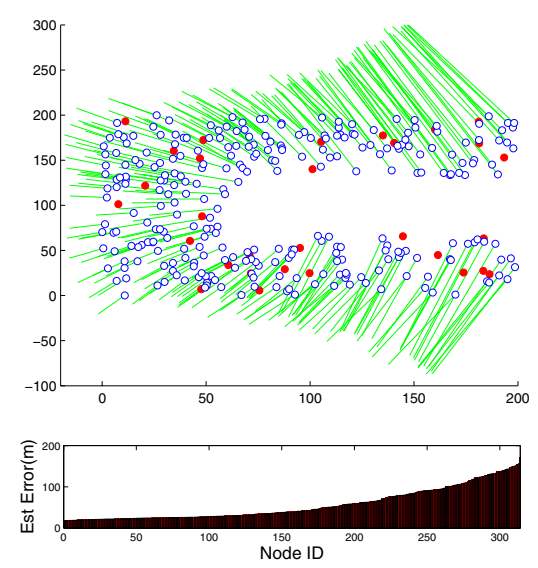

(a) Distance Fitting
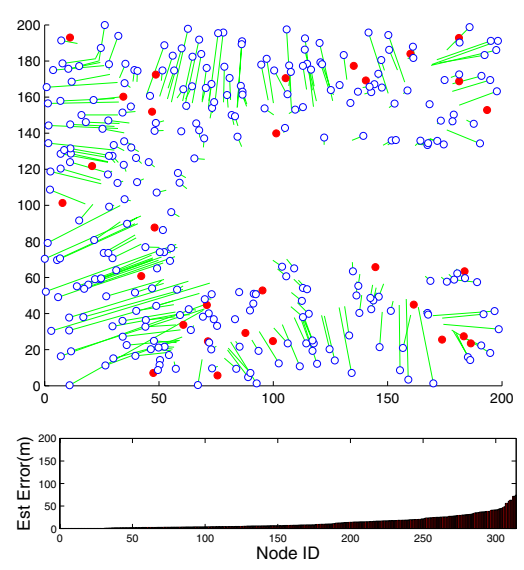

(b) Upper Bound
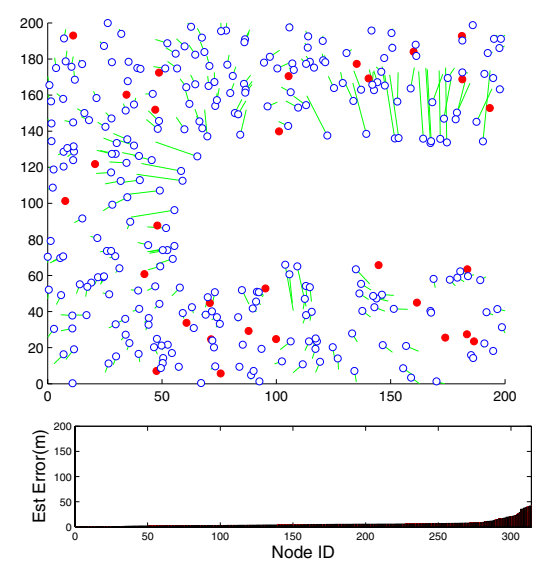

(c) Hybrid

Fig. 7. Comparison of Distance Fitting, Upper bound and Hybrid approaches in C shape configuration

estimated by $\sum_{j \in V_{m}} D_{i j} / \sum_{j \in V_{m}} H_{i j}$ less than the actual one $\sum_{j \in V_{m}} L_{i j} / \sum_{j \in V_{m}}^{j \in V_{m}} H_{i j}$ because some of the distances $D_{i j}$ are much less than the lengths of $L_{i j}$.

To solve the problem above, we need to filter out the pairwise beacon distances which are distorted by concave shapes, which is achievable by reusing the $i$-Multihop algorithm above. Since the calculation of the average length per hop is to infer distances from coordinates, it is a reversed process of localization algorithm which infers coordinates from distances. Therefore, we can use similar idea as $i$-Multihop algorithm to filter out distorted pairwise beacon distances and calculate correct average length per hop as below.

$$
\begin{aligned}
\widehat{l}= & \arg \min _{l} \sum\left(l h_{i}+\varepsilon_{i}-\left|\mathbf{p}_{i}-\mathbf{p}\right|\right)+k \sum \varepsilon_{i} \\
& \text { subject to }\left|\mathbf{p}_{i}-\mathbf{p}\right| \leq l h_{i}+\varepsilon_{i},
\end{aligned}
$$

where $l$ is the average length per hop, $h_{i}$ is the number of hops of the shortest path between $\mathbf{p}$ and $\mathbf{p}_{i}, \varepsilon_{i}$ is the slack variable, and $\mathrm{k}$ is the weight coefficient. The intuitive explanation of the objective function is to find the optimal value of the per hop's average length $l$ which can minimize the difference between the calculated distance $\left|\mathbf{p}_{i}-\mathbf{p}\right|$ and the summary of the measured distance $l h_{i}$ and the slack variable $\varepsilon_{i}$ under the upper bound constraints. Similar to the $i$-Multihop algorithm, with the help of the upper bound constraints, only measured distances which are close to their true Euclidean distances will be involved in the optimization, and the incorrect distance measurements which are much larger than the true values are filtered out. To facilitate the optimization computation, we simplify the objective function above to the following linear optimization.

$$
\widehat{l}=\arg \min _{l}\left(\sum h_{i}\right) l+k \sum \varepsilon_{i}
$$

$$
\text { subject to }\left|\mathbf{p}_{i}-\mathbf{p}\right| \leq l h_{i}+\varepsilon_{i}
$$

\section{Performance evaluation}

We compare the $i$-Multihop algorithm with the original Multihop algorithms and $n$-Multihop algorithm in this section. Since all these three algorithms use the same beacon message flooding or Distance Vector algorithm to compute the number of hops along the shortest paths, the communication cost of the three is the same. Therefore, we can ignore the details of message communication and focus on the character of their geometry calculation. Such an abstraction can help us to evaluate their performance in Matlab simulation, where a sensor network is described as a network graph with vertices representing sensor nodes and edges representing the measurable distances between immediate neighbors.

To investigate the impact of concave shapes on the performance of the Multihop algorithms, we use three basic configurations. In the first configuration, 400 nodes were randomly deployed in a $200 \times 200 \mathrm{~m}^{2}$ square area which has the convex shape. In the second configuration, A portion of sensors in the square area were moved out and the square shape of the network topology is transformed to the $\mathrm{C}$ shape as shown in Fig. 2. In the third configuration, we transform the network topology to the $\mathrm{S}$ shape as shown in Fig. 3.

The following metrics are used in our evaluation.

- Transmission range $R$ : the maximum radio transmission range of sensor nodes.

- Estimation error $\mu_{i}$ : the distance between the estimated position and true position of sensor node $i$, and $\mu_{i}=$ $\left|\widehat{\mathbf{p}}_{i}-\mathbf{p}_{i}\right|$.

- Average estimation error $\widehat{\mu}$ : the average value of the estimation error $\mu_{i}$ and $\widehat{\mu}=\sum \mu_{i} / N$, where $\mathrm{N}$ is the total number of sensors.

\section{A. Comparison of distance fitting, upper bound and hybrid approaches}

We compare the performance of distance fitting, upper bound and hybrid approaches in the $\mathrm{C}$ shape configuration. In this comparison, we assume distance measurements between 


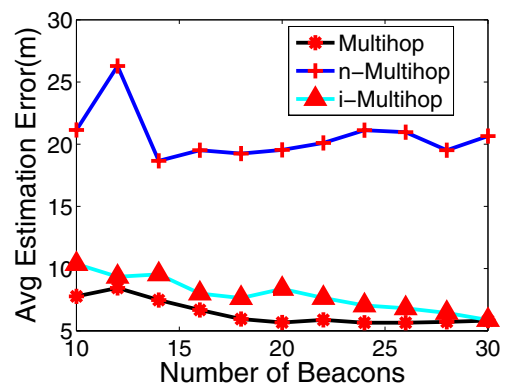

Fig. 8. Square shape configuration

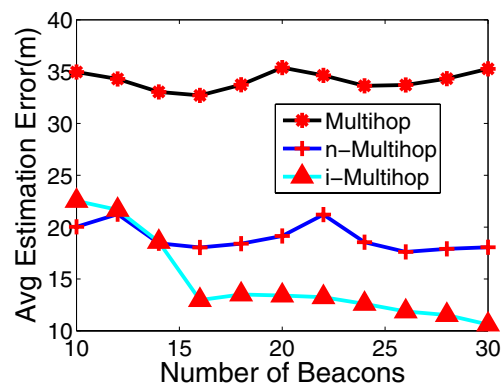

Fig. 9. C shape configuration

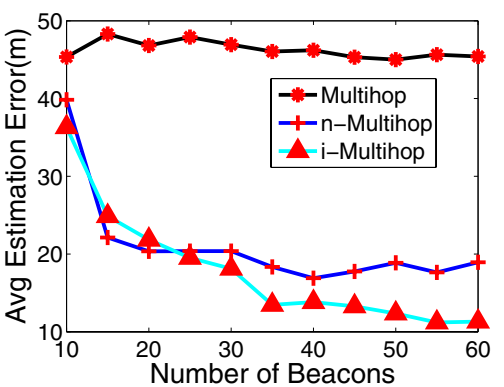

Fig. 10. S shape configuration immediate neighbors are accurate enough, thus the deviation of the shortest path from the straight line is the only error source of distance measurement. The comparison result is shown in Fig. 7, where circles represent the true positions of sensors (solid circles for beacons and empty circles for sensors), and the lines represent the estimation error $\mu_{i}$. The distribution of estimation error $\mu_{i}$ is also described by the bar graph below, where the sensors are ordered by their estimation error $\mu_{i}$. The comparison shows that the distance fitting approach(Fig. 7(a)) has the worst performance, because it tries to fit the distances to all the beacons while some of them are severely distorted by the $\mathrm{C}$ shape. Fig. 7(b) shows that the performance is improved significantly by the upper bound approach, which uses the distance upper bound to filter out the impact of distorted distance measurements. The performance is further improved by the hybrid approach (Fig. 7(c)), which solves the problem that the upper bound approach may have large estimation errors when all the beacons are located in one side of a sensor.

\section{B. Impact of concave shapes}

In this section, we focus the performance comparison on the connectivity-based multihop algorithms, where the distances between immediate neighbors are not available and the average length per hop is sampled from beacons. To investigate the impact of concave shapes on the performance of Multihop algorithms, we compare the Multihop algorithm based on distance fitting, $n$-Multihop algorithm and $i$-Multihop algorithm in square shape, $\mathrm{C}$ shape and $\mathrm{S}$ shape configurations.

Fig. 8 shows the performance comparison of the three algorithms in the square shape configuration. The Multihop and the $i$-Multihop algorithms have similar performance, while the performance of the $n$-Multihop algorithm is much worse than the other two algorithms. The $n$-Multihop algorithm has the worst perform because in connectivity-based multihop algorithm, the distance estimated from the nearest beacon does not guarantee it is the best estimation which is closest to the true Euclidean distance. The performance of the $n$-Multihop algorithm becomes worse when the maximum transmission range becomes much longer than the average distance between immediate neighbors. As we discussed above, the average length per hop $h$ is different from the average distance $d$ between immediate neighbors, and the former is strongly related to the maximum radio transmission range $R$. When the density of deployed sensors is fixed, choosing large transmission range $R$ will improve the network connectivity, which is helpful to sensor localization since the network becomes more rigid with higher connectivity. However, the large transmission range $R$ will lead to the consequence that the average length per hop $h$ is much larger than the average distance $d$ between immediate neighbors. If we estimate distances from the nearest beacon, it is possible that the beacon is within one or two hops range. If it is within one hop range, the true distance to the beacon is close to the average distance $d$ between immediate neighbors. The consequence is that the true distance is less than a single average length per hop $h$, which is smallest measurable unit in connectivity-based Multihop algorithm. This will cause relative large errors from the sensor to nearby beacons. Such a distance estimation error imposes a limitation on the positioning accuracy of the $n$-Multihop algorithm. On the other hand, instead of fitting distances to the nearest beacons, the $i$-Multihop algorithm tries to fit the distance measurements which are closest to their true Euclidean distances, such that the final positioning result of a sensor is closer to its true location.

Fig. 9 shows the performance comparison of the three algorithms in the $\mathrm{C}$ shape configuration. The Mulihop algorithm performs worst, while the $i$-Multihop algorithm is the best of the three. The localization accuracy of the $i$ Multihop algorithm is improved significantly when the number of beacons are increased from 10 to 16, and it eventually converges to a fixed value when the number of beacons is continuously increased. There exists a critical point because in $\mathrm{C}$ shape configuration, when the number of beacons exceeds certain value, most of sensors can have three beacons which are connected by the shortest paths that are close to straight lines. The Mulithop algorithm has the worst performance because some of the distance estimation are distorted by the $\mathrm{C}$ shape. The $n$-Multihop algorithm does not perform as well as the $i$-Multihop algorithm because its localization accuracy is upper bounded by the granularity of the transmission range, as we discussed above.

Fig. 10 shows the performance comparison of the three algorithms in the $\mathrm{S}$ shape configuration, which is more concave than the $\mathrm{C}$ shape and some of the distances estimated by 


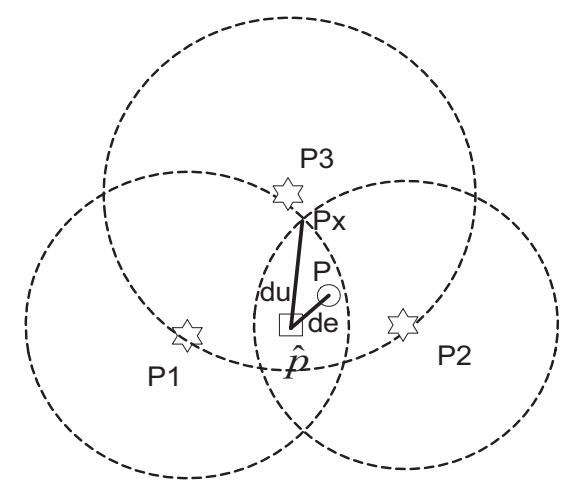

Fig. 11. By its definition, the radius $d_{u}$ is larger than estimation error $d_{e}$

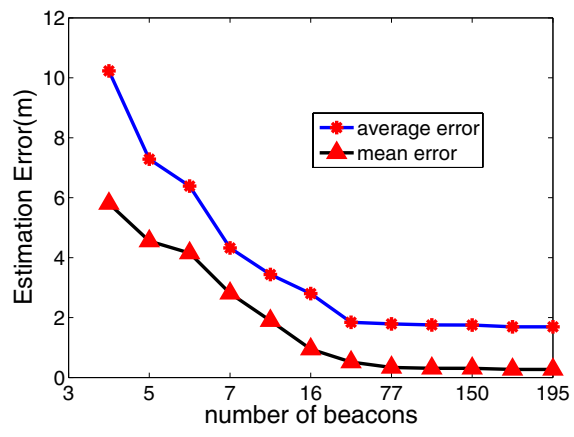

Fig. 12. Positioning accuracy is improved along the iterative process the shortest paths deviate further from their true Euclidean distances. The comparison shows that the Multihop algorithm performs much worse than the $n$-Multihop algorithm and $i$ Multihop algorithm, and the $i$-Multihop algorithm has the best performance. In the $S$ shape configuration, the performance of the $i$-Multihop algorithm is increased significantly when the number of beacons is increased to 30 , and after that, it eventually converges to a fixed value. The critical point of the number of beacons is larger than the $\mathrm{C}$ shape because more beacons are necessary for all sensor to have at least three beacons connected by the close-to-straight-line shortest paths.

From the comparison above we can conclude that the $i$ Multihop algorithm performs best in the three algorithms, and it can locate sensors in concave environments with positioning accuracy comparable to that of convex environments if the number of beacons reaches the threshold.

\section{ITERATIVE APPROACHES}

The simulations above show that certain minimum number of beacons are required for $i$-Multihop algorithm to achieve sufficient localization accuracy in concave areas. More beacons are demanded when deployed areas become more complicated. This is because a sensor can accurately locate itself only when it is connected to at least three beacons by the close-tostraight-line shortest paths. If only a few beacons are deployed in a concave area such as $\mathrm{C}$ shape or $\mathrm{S}$ shape, it is possible that some of sensors are connected to less than three beacons by the close-to-straight-line shortest paths, which results in large estimation errors. In this section, we show that high accuracy can be achieved with less beacons by iteratively applying the $i$-Multihop algorithm.

In the iterative approach, a few beacons are deployed as initial beacons. Due to the small number of initial beacons, they may be "visible" to only small portion of sensors through the close-to-straight-line shortest paths. Those small portion of sensors can accurately locate themselves by referring to the initial beacons. After that, those sensors with accurately determined positions "convert" themselves as new beacons by advertising beacon signals. It is possible that the newly added beacons are connected through the close-to-straightline paths to some sensors which do not have sufficient initial beacons before. By utilizing the beacon signals sent from newly added beacons, those sensors previously with inaccurate estimation results can refine their positions and achieve accurate positioning results. The whole process are recursively repeated until all sensors are accurately located or no more beacons are added.

The challenging of applying the $i$-Multihop algorithm into the iterative process is how to identify "good" candidates which can accurately locate themselves. In other words, we need to estimate how accurate a sensor can locate itself before we can iteratively apply the $i$-Multihop algorithm. Due to the absence of global view of the entire network, a sensor cannot judge if it is connected to at least three beacons through the close-to-straight-line paths. Thus, we cannot identify "good" candidates by simply counting the distances estimated from close-to-straight-line paths. In the following discussion, we propose the upper bound approach to estimate sensors' positioning accuracy.

\section{A. Estimate positioning accuracy}

As we discussed in Section III-C, suppose a sensor $\mathbf{p}$ is within circular region constraints $C_{i}$ with origin $\mathbf{p}_{i}$ and radius $\widehat{d}_{i}$. Here $\mathbf{p}_{i}$ are beacons' positions and $\widehat{d}_{i}$ are estimated distances from $\mathbf{p}$ to $\mathbf{p}_{i}$. Let $S_{t}=\bigcap C_{i}$, which represents the intersection area of all constrained regions $C_{i}$. We notice that the position of the sensor can be pinpointed more accurately when the area of $S_{t}$ becomes smaller. On the other hand, if less than three distance measurements are correct distance estimations, the intersection area $S_{t}$ tends to be large. This observation shows that we can identify good candidate for new beacons by checking the size of the intersection area $S_{t}$.

However, it is difficult to accurately calculate the area of the intersection $S_{t}$ due to its irregular shape. Instead, we use the radius of the intersection $S_{t}$ to estimate the positioning accuracy. As shown in Fig. 11, the intersection's radius $d_{u}$ is defined as the maximum distance between the estimated position $\widehat{\mathbf{p}}$ to any other point $\mathbf{p}_{x}$ within the intersection area and can be calculated as below.

$$
d_{u}=\max _{\mathbf{p}}\left|\widehat{\mathbf{p}}-\mathbf{p}_{x}\right|
$$



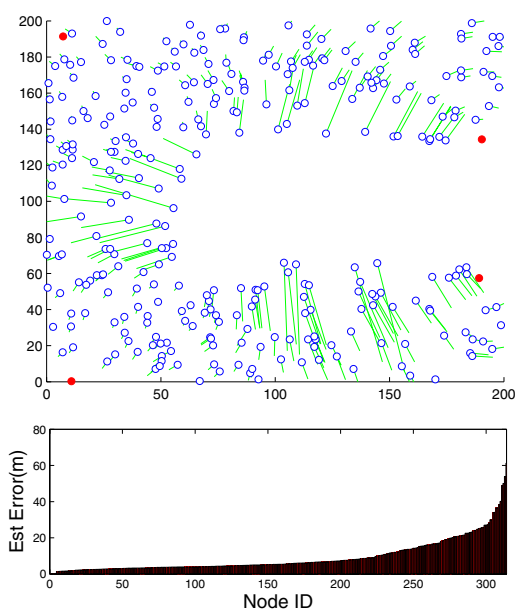

(a) Initial stage
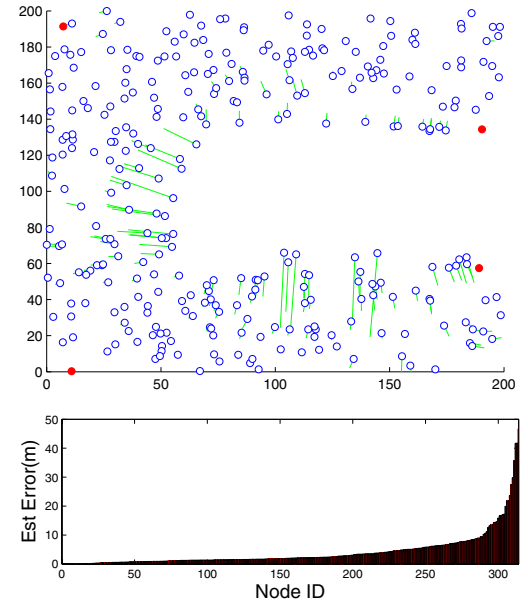

(b) Intermediate stage
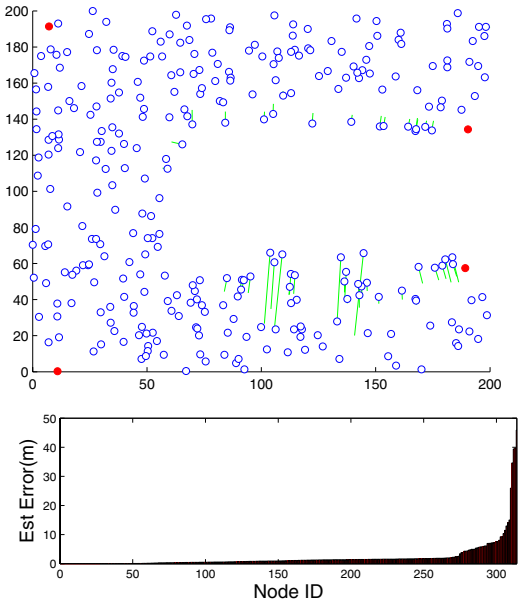

(c) Final stage

Fig. 13. Demo of iterative $i$-Multihop algorithm

$$
\text { subject to }\left|\mathbf{p}_{x}-\mathbf{p}_{i}\right| \leq \widehat{d}_{i}
$$

Here, $\widehat{\mathbf{p}}$ is the position estimated by our $i$-Multihop algorithm, $\mathbf{p}_{i}$ are beacons' positions and $\widehat{d}_{i}$ are measured distances. By the definition of $d_{u}$, we have $d_{u} \geq d_{e}$, where $d_{e}$ is the estimation error between estimated position $\widehat{\mathbf{p}}$ and the true position p. In other words, $d_{u}$ is the upper bound of the estimation error $d_{e}$ and can be used to estimate the positioning accuracy. Sensors with small estimation errors are identified if they have small estimation radius $d_{u}$.

\section{B. Apply i-Multihop algorithm iteratively}

Based on the radius $d_{u}$, we can find out sensors with high positioning accuracy, which makes it possible to apply $i$-Multihop algorithm iteratively in concave areas. In the iterative approach, positions of beacon nodes are broadcasted through beacon signals. Beacon signals have counters which are increased by the lengths of hops when they are forwarded between neighboring sensors. The length of the shortest path from a sensor to a beacon can be found out from the minimum counter value among all the received beacon signals sent out by that beacon. Therefore, each sensor can learn beacons' positions and the lengths of the shortest paths to those beacons. Sensors keep listening to beacon signals and refine their positions each time when new beacon signals are received. At the same time, sensors' position accuracy is estimated by their radiuses $d_{u}$. When the radius of a sensor is less than the threshold, it will advertise itself as a new beacon, whose beacon signals can be utilized by other sensor to refine their estimated positions. The positioning processes are repeated until all sensors are accurately located or no more beacons are added. Note the iterative process can be achieved by sensors localized computation which consists of beacon signals listening and position refining. Therefore, it can be implemented in a fully distributed fashion.

\section{Performance of iterative i-Multihop algorithm}

We evaluate the performance of the iterative $i$-Multihop algorithm as following. In the evaluation, 314 nodes are deployed in a $\mathrm{C}$ shape area with only 4 initial beacons are deployed at the four corners. We assume that distances between neighboring sensors are measurable, thus the mismatch between the shortest path and the straight line is the main source of the distance measurement error. Fig. 12 shows both the average estimation error and the mean estimation error are decreased along the iterative process when more and more beacons are involved in the localization process. We also note that the mean estimation error is always smaller than the the average estimation error. This is because a small portion of sensors have much larger errors than the rest of sensors. To further illustrate the iterative $i$-Multihop algorithm, an example is shown in Fig. 13. At the beginning(Fig.13(a)) when only the four initial beacons are used, a number of sensors have large errors because they do not have three beacons visible through the close-to-straight-line shortest paths. Fig. 13(b) shows the intermediate status of the iterative process, where some sensors improve their positioning accuracy by referring to newly added beacons. Fig. 13(c) shows the final stage of the iterative process, where majority of sensors can locate themselves accurately. We notice that there are a few sensors which can not locate themselves accurately in the final stage. This is because those sensors do not have good beacons' layout even with the help of iterative approach. Those sensors with large estimation errors can be identified by their radius $d_{u}$, thus we can notify upper layer location-aided applications when sensors have large estimation errors and the positioning results are unreliable.

The iterative $i$-Multihop algorithm differs from previously proposed iterative approaches in the following aspects:

- It does not require that initial beacons are adjacent to each other, which is an implicit assumption for other iterative 
approaches to initiate the iterative process.

- For beacons to be propagated to entire areas, previous iterative approaches usually require dense and uniform sensor distribution. Otherwise, the newly added beacons cannot approach some sensors and the whole iterative process is interrupted. On the contrary, in the iterative $i$ Multihop algorithm, all sensors can be located as long as they form a connected network. This is because sensors' positions are first estimated from initial beacons, and then refined by newly joined beacons. Here, the iterative strategy are mainly used to improve the positioning accuracy.

- If the positioning accuracy cannot be improved by the iterative process due to awkward beacon layout, the positioning error can be estimated by the radius $d_{u}$, which can be sent to upper layer applications together with the positioning data. With the notification of positioning accuracy, the upper layer location-aided applications can utilize the location information more intelligently by prudently dealing with the sensors with large estimation errors.

- A potential problem of previous iterative approach is that the positioning errors may accumulate along the iterative process. This is because the newly joined beacon are not as accurate as initial beacons and may have large estimation errors, especially the flip-over errors discussed in [12]. The estimation errors of newly joined beacons may accumulate in the following localization process and the final results are severely corrupted. The accumulative errors are minimized in iterative $i$-Multihop algorithm because of two reasons: (1) We use the radius $d_{u}$ to estimate positioning accuracy and beacons are only converted from sensors which can accurately locate themselves. (2) In the iterative $i$-Multihop algorithm, the positioning accuracy is consistently increased because sensors' estimated positions are updated by new beacon signals only when their estimation accuracy are improved, i.e. smaller radius $d_{u}$ can be achieved.

\section{CONCLUSION}

In this paper, we investigate the Multihop algorithms in concave environments, where some of the distances estimated from the shorted path significantly deviate from the true Euclidean distances. While previous approaches cannot perform well due to distorted distance estimation, our proposed $i$-Multihop can filter out the impact of distance measurements with large errors and achieve sufficient localization accuracy. Therefore, we extend the original Multihop algorithm to concave environments, which is necessary in many cases when sensors are deployed in either urban areas or wild habitats. We also show that the $i$-Multihop algorithm can be iteratively applied to achieve accurate localization results with less beacons. Moreover, the $i$-Multihop algorithm can estimate the positioning accuracy, which provides a good basis for upper layer application to intelligently utilize the location data.

\section{ACKNOWLEDGMENT}

The authors would like to thank the anonymous referees for their critical and constructive comments on this paper. They would also like to thank Ruth Mendel for reading the paper and her suggestions. This work is supported in part by the US National Science Foundation under grants CCF-0325760, CCF 0514078, and CNS 0549006.

\section{REFERENCES}

[1] B. Karp and H. T. Kung, "GPSR: Greedy Perimeter Stateless Routing for Wireless Networks," in MobiCom, 2000.

[2] Y. Ko and N. H. Vaidya, "Location-aided routing (LAR) in mobile ad hoc networks," in MobiCom, 2000.

[3] J. C. Navas and T. Imielinski, "Geographic addressing and routing," in MobiCom, 1997.

[4] G. Simon, M. Marti, A. Ldeczi, G. Balogh, B. Kusy, A. Ndas, G. Pap, J. Sallai, and K. Frampton, "Sensor Network-Based Countersniper System," in SenSys, 2004.

[5] Y. Shang and W. Ruml, "Improved MDS-Based Localization," in $I N$ FOCOM, 2004.

[6] H. Lim and J. C. Hou, "Localization for anisotropic sensor networks," in InfoCom, 2005.

[7] A. Savvides, C. Han, and M. B. Strivastava, "Dynamic fine-grained localization in ad-hoc networks of sensors," in MobiCom, 2001.

[8] K. Whitehouse, "The design of calamari: an ad-hoc localization system for sensor networks," Master's Thesis, University of California at Berkeley, 2002.

[9] D. Niculescu and B. Nath, "Ad hoc Positioning System (APS)," in GLOBECOM, 2001

[10] T. He, C. Huang, B. M. Blum, J. A. Stankovic, and T. F. Abdelzaher, "Range-Free Localization Schemes in Large Scale Sensor Networks," in MobiCom, 2003.

[11] J. Albowicz, A. Chen, and L. Zhang, "Recursive Position Estimation in Sensor Networks," in ICNP, 2001.

[12] D. Moore, J. Leonard, D. Rus, and S. Teller, "Robust Distributed Network Localization with Noisy Range Measurements," in SenSys, 2004.

[13] R. Nagpal, H. Shrobe, and J. Bachrach, "Organizing a Global Coordinate System from Local Information on an Ad Hoc Sensor Network," in IPSN, 2003

[14] A. Savvides, H. Park, and M. B. Srivastava, "The n-hop multilateration primitive for node localization problems," in Mobile Networks and Applications, 2003.

[15] A. Savvides, H. Park, and M. B. Srivastava, "The bits and flops of the nhop multilateration primitive for node localization problems," in WSNA, 2002.

[16] Y. Shang, W. Ruml, Y. Zhang, and M. P. J. Fromherz, "Localization from mere connectivity," in MobiHoc, 2003.

[17] X. Ji and H. Zha, "Sensor Positioning in Wireless Ad-hoc Sensor Networks with Multidimensional Scaling," in INFOCOM, 2004.

[18] L. Doherty, K. S. J. Pister, and L. E. Ghaoui, "Convex Position Estimation in Wireless Sensor Networks," in INFOCOM, 2001.

[19] P. Biswas and Y. Ye, "Semidefinite Programming for Ad Hoc Wireless Sensor Network Localization," in IPSN, 2004.

[20] Y. Shang, H. Shi, and A. Ahmed, "Performance tradeoffs of localization methods in ad-hoc sensor networks," in MASS, 2004.

[21] N. Li and J. C. Hou, "Flss: A fault-tolerant topology control algorithm for wireless networks," in MobiCom, 2004.

[22] X.-Y. Li, P.-J. Wan, Y. Wang, and C.-W. Yi, "Fault tolerant deployment and topology control for wireless ad hoc networks," in MobiHoc, 2003.

[23] C. Wang, L. Xiao, and J. Rong, "Sensor localization in an obstructed environment," in DCOSS, 2005. 